\title{
The Simulation of Vessel Traffic Flow Based on Congruential Generator
}

\author{
Liu Zheng-bin, Fu Yu-hui, Cong Ye-sheng \\ Dalian Maritime University, Dalian, China
}

\begin{abstract}
In order to adapt the rapid development of port, to solve relative problems, this paper made a fundamental simulation of vessel traffic flow. Based on the congruential technology, combined with vessel traffic flow, vessel speed, interval between ships and ship turning information, the Ship Produce Model was built. And the ship initial information system was made. It is concluded that this model is easy to understand and operate. It also can provide some parameter support to study the encounter problems and so on.
\end{abstract}

Key words: Vessel traffic volume; Vessel speed; Interval between ships; Random number; Ship Produce Model

Actually the work of this paper is to establish the ship generated model and simulation. Substantially, generation model of the ship influence the input part of the role of the traffic flow on the beginning of the simulation fairway section, and relies on random number technology that meets the given parameters of the probability distribution of vessel traffic flow as well as the ship's state property. Generated model of the ship is the beginning of the simulation of the traffic flow, but also is to establish a fundamental part of the traffic flow simulation model to produce the initial information which is enough to fit realistic generated model of the ship, namely transport stream input which supports scientific analog security for the late running of the ship model.

The traffic flow information in the generated model of the ship reflects the randomness of the system initialization - the randomness of the waterway starting section ship arrival times. On the basis of the analysis of actual ship information about the AIS records during the simulation, choosing the traffic volume, speed and distance between ships, as well as assumptions of ship steering as dynamic initial information about traffic flow. According to the traffic volume to determine the number of generated ships, the speed distribution to determine the initial speed, the distance between ships to determine the initial moment of the ships, the steering information to determine the direction of the next turning point.

\section{Uniform Random Number}

During the establishment of the modeling process, in order to fully demonstrate the randomness of the vessel traffic flow to ensure the credibility of the simulation, therefore, the method of generating random numbers becomes the crux of the final simulation. The random number is not an exact definition, from the general sense, is a group of deterministic, unpredictable sequence of numbers. Judging the quality of the sequence of random numbers consists of three indicators relevance, cyclical and distribution characteristics.

The random number in this article is the uniformly distributed random number, which obeys the $\mathrm{U}(0,1)$ distribution. After generating this random number, it can be generated by a certain method to obey other random distribution of the random variable. Thereby, it performs effective simulation.

Congruential generators are important technical methods of producing a uniform distribution random number, mainly including linear congruential generator, model and mixed linear congruential generator and congruential generator of prime numbers. According to the specific needs of the actual computing power of the computer model, this article uses a linear congruential generator to produce uniformly distributed random numbers. Its general recursive formula is:

$$
\left\{\begin{array}{l}
x_{n}=\left(a x_{n-1}+c\right)(\bmod M) \\
r_{n}=x_{n} / M \\
x_{0}
\end{array} \quad(n=1,2, \cdots)\right.
$$

Tab. 1 Relationship between traffic volume and frequency Where, $a$ is multiplier, $c$ is addend, $x_{0}$ is initial value, $M$ is 
mode. Moreover, $x_{\mathrm{n}}, a, c, M$ are four parameters which are

\begin{tabular}{|cc|}
\hline Traffic Volume & Frequency \\
\hline 1 & 1 \\
\hline 2 & 2 \\
\hline 3 & 3 \\
\hline 4 & 5 \\
\hline 5 & 1 \\
\hline 6 & 3 \\
\hline 7 & 4 \\
\hline 8 & 3 \\
\hline 9 & 1 \\
\hline 10 & 3 \\
\hline 11 & 1 \\
\hline 12 & 1 \\
\hline
\end{tabular}

non-negative integers. According to formula, $x_{0}$ is compatible with $0 \leqslant x_{0}<M$ and derivates $0 \leqslant r_{n} \leqslant 1$. It will get a set of uniform random numbers. However, selecting four parameters is most important to the quality of random number. According to personal experience, $M, a, x_{0}, c$ equals to $256,5,8,17$ respectively in this article.

\section{Random Distribution of Traffic Flow Parameters}

In this article, the choice of a certain waters which is at 0:00-1:00 on May 4 to May 31 as records all information of ship which goes to north, and organize and analyze these ship information to acquire the relevant characteristics of the traffic flow parameters.

\subsection{Vessel Traffic Volume}

Collating of all data, it could be concluded that the minimum and maximum values of traffic volume are 1 and 12 , observed frequency of 28 times. Traffic volume and the frequency relationship is shown in Table 1.

Using the following method to simulate the traffic volume, the statistics number shows there are totally 28 times observational data. From theTab.1, the ship number of once appearance is $1,5,9,11$ and 12 which is totally 5 times; the ship number of twice appearance is 2 which is totally 2 times; the ship number of third appearance is $3,6,8$ and 10 which is totally 12 times; the ship number of forth appearance is 4 which is totally 4 times; the ship number of fifth appearance is 5 which is totally 5 times; combine the ship number which ship has appeared five times, totally 10 times.

Therefore let the random variable $X=0$ indicates the number of vessels occurs 2 times, $X=1$ indicates the number of vessels occurs 4 times, $X=2$ indicates the number of vessels occurs 10 times, $X=3$ indicates the number of vessels occurs 12 times. Assuming its probability is $\mathrm{p}_{1} 、 \mathrm{p}_{2} 、 \mathrm{p}_{3}$ and $\mathrm{p}_{4}$, and its value

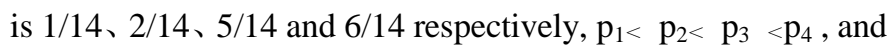
$\mathrm{p}_{1}+\mathrm{p}_{2}+\mathrm{p}_{3}+\mathrm{p}_{4}=1$. It generates a random number $\mathrm{u}_{1}$ which obey the distribution of $\mathrm{U}(0,1)$. If $0<\mathrm{u}_{1} \leqslant \mathrm{p}_{1}, \mathrm{X}=0$ which means to generate two ships; if $\mathrm{p}_{1}<\mathrm{u}_{1} \leqslant \mathrm{p}_{1}+\mathrm{p}_{2}, \mathrm{X}=1$ which means to generate four ships; if $\mathrm{p}_{1}+\mathrm{p}_{2}<\mathrm{u}_{1} \leqslant \mathrm{p}_{1}+\mathrm{p}_{2}+\mathrm{p}_{3}, \mathrm{X}=2$ means which the generation of ship numbers may be one, five, nine, eleven, twelve, or four. Due to the existence of merge term, it should be further processed. For $X=2$, generate an equal probability random number $\mathrm{u}_{2}$ among the random event $\mathrm{Y}=0$ 、 1 、 2、 3 which all of the value of probability are 0.4 , and the generated corresponding ship number is $3,6,8,10$; for $X=3$, assume the random variable $\mathrm{P}=0 、 1$ and the value of probability is $\mathrm{p}_{1}=1 / 6$ and $\mathrm{p}_{2}=5 / 6$ which generate the random number obey $\mathrm{U}(0,1)$. If $0<\mathrm{u}_{3} \leqslant \mathrm{p}_{1}, \mathrm{P}=0$ which the generation of ship number may be $1,5,9,11$, or 12 ; if $\mathrm{p}_{1}<\mathrm{u}_{3} \leqslant 1, \mathrm{P}=1$ which the generation of ship number is 5 . Due to the existence of merge term, it also should further process $\mathrm{P}=0$. Same as above, generate an equal probability random number $\mathrm{u}_{4}$ among the random event $\mathrm{Q}=0 、 1 、 2 、 3 、 4$ which all of the value of probability are 0.2 , and the generated corresponding ship number is $1 、 5 、 9 、 11 、 12$.

\subsection{Speed}

According to statistics, there are 177 speed values which the minimum and maximum are 6.2 and 17.1 respectively. To find out $\mu$ of these values and $\sigma^{2}$ of the maximum likelihood estimator, $\quad \mu=x=11.1 \quad, \quad \sigma^{2}=\frac{1}{177} \sum_{i=1}^{177}\left(x_{\mathrm{i}}-x\right)^{2}=$ 3.086, $\sigma=\sqrt{3.086}=1.76$, and the $\chi^{2}$ test is showed in Table 2.

Where $\mathrm{n}=180$. The value of $r, k 、 \alpha$ is $6 、 2$ and 0.05 respectively, so $\chi_{0.95}^{2}(6-2-1)=\chi_{0.95}^{2}(3)=7.815$, $3.055<7.815$. Thus, sample is normal distribution which average is 11.1 and the variance is 1.76 . 
Tab. 2 Process of speed data

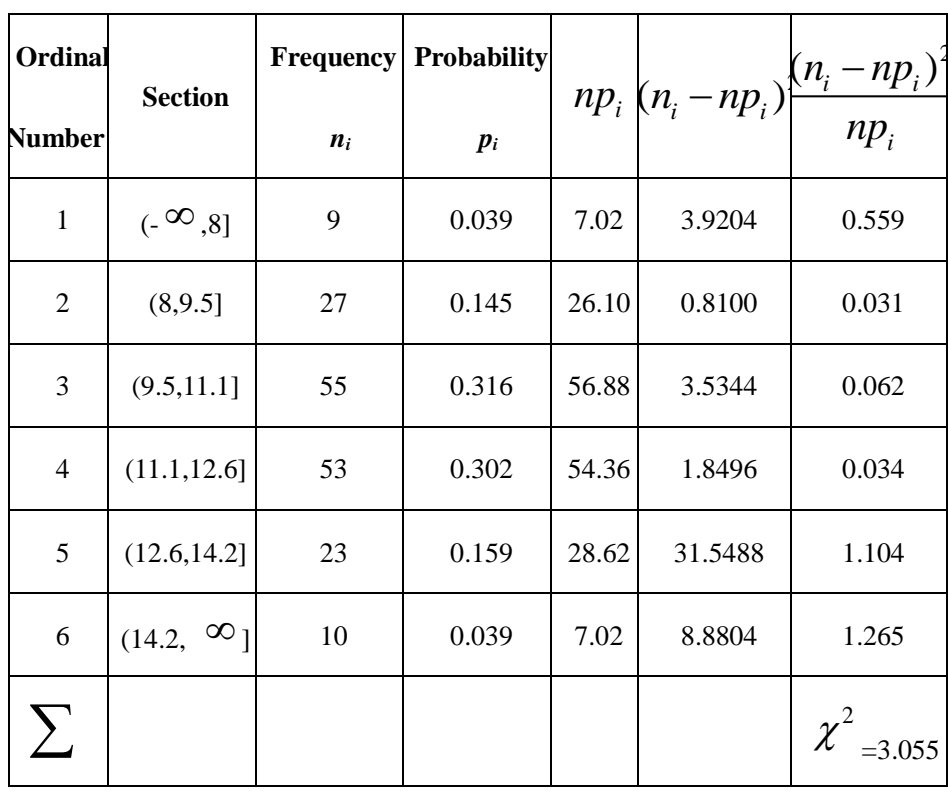

Normal distribution function could not gain display format through the integral. Therefore, the Box-Muller function transformation method be used and the instruction of method is that we know that when a random variable $X$ obeys the standard normal distribution $N(0,1)$, the random variable $Y=\sigma X+\mu$ will obey normal distribution $N\left(\mu, \sigma^{2}\right)$. So, first, we can generate two random number $\mathrm{u}_{1}$ and $\mathrm{u}_{2}$ which obey uniform distribution and through the transformation to get random variable $W_{1}$ and $W_{2}$ to make them obey the standard normal distribution.

\subsection{The Time-distance Between Ships}

First, the data for all 28 days of the ship between 0:00 to 1:00 which using one minute as interval time to be organized. The organized data are the ship volume which arrives the observation point in per minute. The relation of time and ship volume is shown in Table 3.

Using MATLAB curve fitting toolbox to get time and ship volume obeys exponential distribution, fitting formula is $f(x)=a e^{b x}$. If using the $95 \%$ confidence interval, the coefficients value of the model $\mathrm{a}$ and $\mathrm{b}$ are $1.639 \times 10^{-8}$ and 0.3043 respectively.

Reserve transformation of exponential function could be $x=(1 / b) \ln (u / a)$ and using $\mathrm{U}(0,1)$ to get a random number which be plugged into equations to get the departure time of the vessel. However, if the time value less than thirty-five and more than fifty-nine, it would be seen as invalid value. In that situation, it should generate new random numbers and recalculate time value.
Tab. 3 Relationship between time and ship

\begin{tabular}{|c|c|c|c|}
\hline Time/minute & Ship Volume & Time/minute & Ship Volume \\
\hline 35 & 0 & 48 & 2 \\
\hline 36 & 0 & 49 & 5 \\
\hline 37 & 0 & 50 & 9 \\
\hline 38 & 1 & 51 & 2 \\
\hline 39 & 0 & 52 & 5 \\
\hline 40 & 0 & 53 & 7 \\
\hline 41 & 1 & 54 & 14 \\
\hline 42 & 1 & 55 & 17 \\
\hline 43 & 0 & 56 & 16 \\
\hline 44 & 0 & 57 & 18 \\
\hline 45 & 0 & 58 & 19 \\
\hline 46 & 1 & 59 & 55 \\
\hline 47 & 1 & & \\
\hline
\end{tabular}

\subsection{Steering}

There is no steering information on the next ship turning point in AIS information, but the destination of shipping is certain when departure, so this article assumes the existence of steering information based on the actual situation. The method used in this paper assumes that there are three driving directions for ship turning in the next corner which are turn left, go straight and turn right. This assumption is in line with the actual situation. Next, using a random event $\mathrm{X}$ means turning left, going straight and turning right to the occurrence of the corresponding event, so that $\mathrm{X}=0$ indicates that the ship turns left at the next corner, $X=1$ represents that the ship goes straight at the next corner, and $X=2$ said that the ship turns right at the next corner. At the same time, it is assumed that the proportion of ship turning left, going straight and turning right as $\mathrm{p}_{1} 、 \mathrm{p}_{2}$ and $\mathrm{p}_{3}$, and the relationship is $\mathrm{p}_{1}<\mathrm{p}_{2}<\mathrm{p}_{3}$ (the size relations of three variables are uncertain and it is only a hypothesis), moreover, $\mathrm{p}_{1}+\mathrm{p}_{2}+\mathrm{p}_{3}=1$.

\section{Model Simulation}

Using access data base tool to simulate and VHB program language to write the computer program, we can get the 
inputting the interface of human-computer interaction .In the ship's initial information generating system, when we input every parameter of the distribution function information that has been counted out, inputting 11.1 and 1.76 into the speed columns respectively, inputting 0.00000001639 and 0.3043 into the time column respectively, inputting $0.5,0.2$ and 0.3 into the direction of probability value column respectively, inputting 256, 5, 8, 17 into the random number column, click on the information generated button, we can get randomly generated ship initial information. Then we put the generated information in an Excel table, as showed in figure1.

\begin{tabular}{|c|c|c|c|c|}
\hline A & B & C & D & E \\
\hline Serial Number & Ship's Number & Speed & Time & Direction \\
\hline 1 & Ship 1 & 16.11 & 56 & RIGHT \\
\hline 2 & Ship 2 & 15.27 & 56 & LEFT \\
\hline 3 & Ship 3 & 10.26 & 56 & RIGHT \\
\hline 4 & Ship 4 & 6.46 & 59 & LEFT \\
\hline 5 & Ship 5 & 8.75 & 59 & LEFT \\
\hline 6 & Ship 6 & 11.88 & 59 & LEFT \\
\hline 7 & Ship 7 & 9.64 & 59 & RIGHT \\
\hline 8 & Ship 8 & 19.54 & 59 & LEFT \\
\hline 9 & Ship 9 & 7.4 & 59 & STRAIGHT \\
\hline 10 & Ship 10 & 22.86 & 59 & STRAIGHT \\
\hline 11 & Ship 11 & 8.49 & 59 & STRAIGHT \\
\hline 12 & Ship 12 & 9 & 59 & LEFT \\
\hline
\end{tabular}

Fig.1 Table of ship initial information

Taking each generation process from individual ship initial information into account, we can get the result that the traffic volume is between 1 and 12, the minimum value is 1 and the maximum value is 12 , which accords with the random judgment we made on the basis of statistics; the minimum speed value is 2.74 and the maximum value is 33.78 , which conforms to the actual vessel speed possible value and are also in statistical velocity value range; each value is in agreement with clock timing; In direction term, it will appear left, straight, right three directions.

Looking 50 times generation information , 271 ship initial information from the whole, each traffic volume accords with probability value and statistics; using rounding off method to get speed value accurate to a decimal point, again we make velocity analysis, and then we get estimated mean speed which is 15.5 and estimated mean variance which is 1.85 .The values are approximate values with foregoing values, the minimum value is 2.74 , the maximum value is 38.73 , there appears 9 times that the value is less than 5 and 9 times that is larger than 30 and smaller than 38.73 , two value of the probability is 0.033 .
Although there are nine speed values much bigger, the values accords with the actual situation in some extent. As time aspect, most of the values fall in between 55 and 59 which accords with time distribution. As for the direction information, in the 271 message, there appear 157 left turns, 54 straight, 60 right turns. The probability of the three is $0.58,0.20,0.22$, which is almost the same with the simulation inputting.

\section{Result and Forecast}

This paper use characteristics of vessel traffic flow parameters and generating random number which obey evenly distributing as the foundation to establishing ship generation model. We have taken parameter characteristics of actual vessel traffic, ship speed and time distance between ships into consideration and put forward a veer hypothesis. In the end, we write a program to simulate the ship generation model. The results show that this model is easy to understand and easy to implement. It also has certain reliability in the ship's initial information generation.

In the later work, we can improve the random number generation techniques and use more reasonable and effective random number technology. Furthermore, the model time could be longer. In data acquisition, we can choose the proper time which has a larger traffic volume to finish the analysis and take the ship individual information into the model for consideration to improve ship generation model.

\section{References}

[1] Wu Zhao-ling and Zhu Jun, Marine Traffic Engineering, $2^{\text {nd }}$ ed, DLMU, 2004.

[2] Jin Wen-zhou, Zhang Jie and Zhen Ying-li, 'Traffic Flow Simulation Model Based on Cellular Automata Theory', in Natural Science Eds.Vol.29(3). South China University of Technology: Academic, 2001, pp.93-96.

[3] Shao Chang-feng and Fang Xiang-ling, 'Fluid Model of The Vessel Traffic Flow',Vol.28(1). DLMU: Academic, 2002, pp.52-55.

[4] Yang Hong-li and Bao Cheng-you, Probability Theory and Mathematical Statistic, BUPT: Academic, 2007.

[5] Jun Zhi-cai, Modeling and Simulation of Transportation Systems, Beijing: Science Eds, 2011.

[6] Li Li, Jiang Rui and Jia Bin, The Theory and Application of Modern Traffic Flow, Vol.1, Beijing: Tsinghua University Eds, 2011. 